\title{
AFV Vetronics: Displays Design Criteria
}

\author{
Branko Livada 1 - Radomir Janković2 - Nebojša Nikolić3 \\ ${ }^{1}$ Luxell Technologies Ltd., Canada \\ 2 Union University School of Computing, Serbia \\ ${ }^{3}$ Strategic Research Institute, Serbia
}

\begin{abstract}
This paper addresses the basic functionalities and properties of displays aimed to be used in modern armoured fighting vehicles (AFV). The paper also establishes a basis to determine the opportunities for a different technology application in the harsh AFV environment. The AFV displays are specified by active area, footprint size and other characteristics such as luminance, resolution, viewing envelope, colour, grey scale, night vision compatibility, and sun light readability. Also, some specific requirements for AFV display properties related to AFV application environment are discussed. This paper further provides a short overview of the key technologies used in display design and their compliance with basic AFV requirements. After ruggedisation of the Commercial Off The Shelf - COTS AMLCD flat panels have been successfully applied, they obtained dominant application in AFV displays. It seems that AMLCD display application in AFV will dominate during this decade.
\end{abstract}

Keywords: vetronics, AFV displays, Ruggedised displays, AMLCD technology, flat panel displays

\section{O INTRODUCTION}

Recent improvements in information and armoured fighting vehicles (AFV) technologies have introduced new directions in development of electronic devices for vehicle control and a new tactical approach for the use of AFVs in battlefields.

Vetronics (Vehicle electronics) is becoming a key feature of both new AFV developments and upgrades of existing vehicles. In general, the architecture of the vetronics system includes some of functional subsystems:

- fire control system,

- vehicle control,

- active suspension,

- engine control and monitoring,

- sensors control and signal distribution,

- data collection and distribution systems,

- communication systems.

AFV upgrade projects could use all or only some of the sub-systems listed.

The brisk development of information technology has changed the way warfare is conducted, which is expressed in the so called Network Centric Warfare (NWC) doctrine [1] and [2] and swarming [3] and [4] as a new tactical approach.

In spite of almost 100 years of their deployment in active service, AFV still are one among the most important resources of every contemporary army's ground forces. Contemporary warfare has significantly changed the way of armed forces use, resulting in the need for research and introduction of new tactical procedures, wherein swarming is the most promising one. In the military sense of the word, swarming [3] is " . .. a systematic pulsing of force and/ or by dispersed networked units, so as to strike from all directions simultaneously." An example of AFV swarming tactics is depicted in Fig. 1

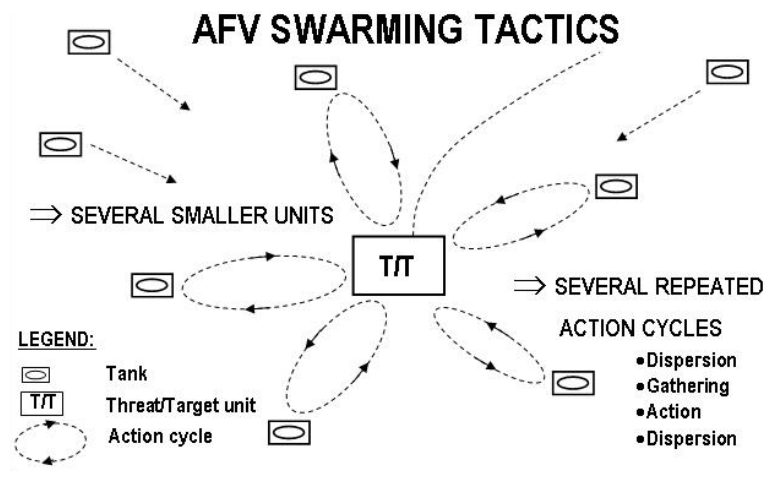

Fig. 1. AFV swarming tactics

In order to enable AFVs (main battle tanks, APCs, scout vehicles etc.) acting as swarmers, it is necessary to develop an appropriate C4ISR system [4] and [5] centred on computers, navigation and displays for crew interaction.

The display is one of the key components of ${ }^{4}$ ISR system, being an integrated piece of equipment for AFV crew access to all information necessary to control motion and actions of their AFV. It enables them to take part in common actions of all AFVs applying swarming tactics: controlled motion and actions within the swarm, and active participation in ${ }^{4}$ ISR system, both as the information source and recipient.

This paper discusses various display technologies, and how their capabilities could be used in AFV vetronics and AFV Crew Station design. 


\section{AFV VETRONICS SYSTEM STRUCTURE AND REQUIREMENTS}

AFV vetronics system architectures are still under development [10] to [13]. The key activities are in the field of interface developments and related software developments. Some development results are already applied in new and upgraded AFV projects.

A generalized AFV vetronics system is illustrated in Fig. 2. AFV vetronics architecture tends to integrate vehicle power, physical properties, control software, data transfer and human factors.

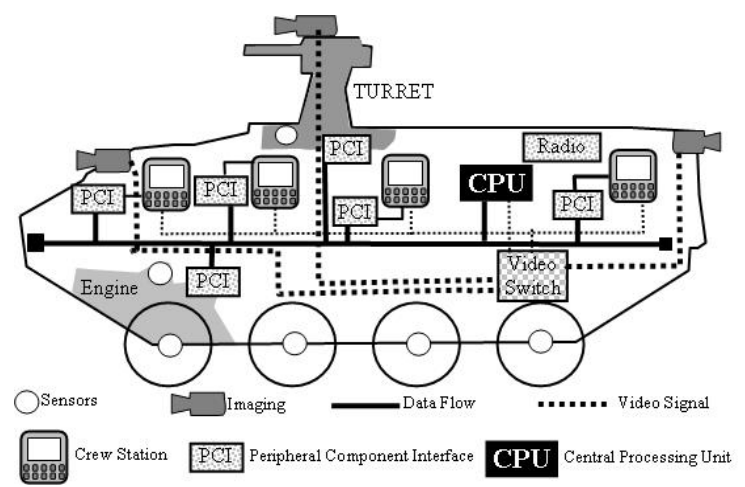

Fig. 2. AFV vetronics generalized architecture

AFVs operate in extreme climatic conditions, and generate harsh mechanical influences. Climatic environmental conditions are usually analyzed and related design requirements are defined using STANAG 2895 [6]. Environmental compliance is usually verified by using MIL STD 810 [7].

Modern AFV displays typically provide the following:

- function keys - providing customization of the displayed information,

- text and graphic overlay allowing customized HMI (Human Machine Interface) for individual application (reticules, icons, menus etc.),

- picture in picture presentation,

- connectivity (CANbus, MilCAN, serial RS232, RS422, USB, wireless);

- video (analogue NTSC, CCIR RS170, and/or digital DVI),

- recording - to be applied as per specific operations and training purposes.

Bearing in mind these requirements, the display workload can be divided into various categories:

- MFD - multi-functional displays,

- VD - video displays,

- MMD - moving map displays,

- HUMS - health \& usage monitoring station,
- ISD - individual sensor displays.

MFD are usually applied at commander and/or Gunner Control Station. VD and HUMS are usually applied at driver and commander control station. ISD could be applied at drivers control station, and anywhere needed.

A block diagram of typical AVF crew station is depicted in Fig. 3.

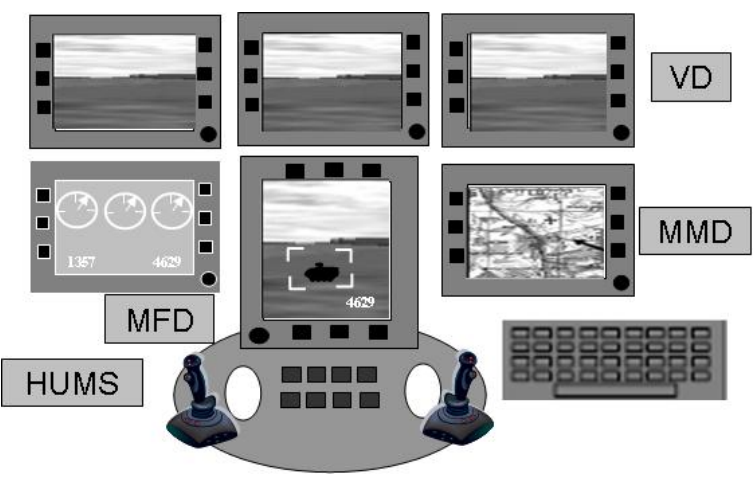

Fig. 3. AFV crew station block diagram

\section{RUGGEDISED DISPLAY STRUCTURE}

Whenever there is a need to obtain some additional display feature for specific application (wide temperature range \& harsh environmental influences, high reliability and long lifetime, high ambient illumination readability, etc.) one should ask for a ruggedised display solution [14]. Depending on the application, different levels of ruggedisation and appropriate technical solutions can be applied [15].

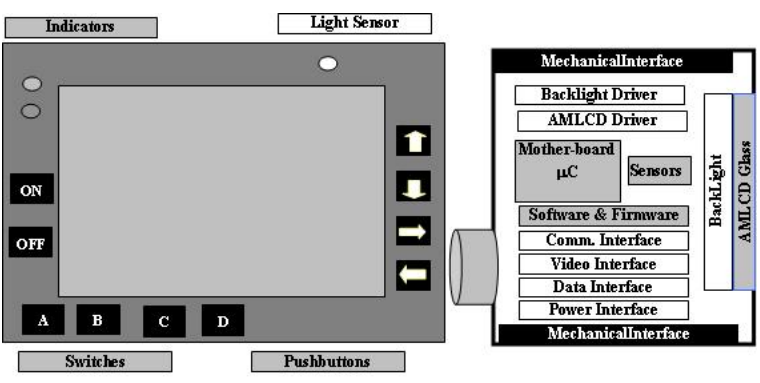

Fig. 4. AFV ruggedised display structure

In the present time, preferred solutions use COTS (Commercial off the Shelf) AMLCD panels ruggedised to work properly for specific applications [9]. That adaptation process and/or ruggedisation usually involve the following engineering solutions and changes:

Mechanical design: contributes to the display protection from all the specified environmental conditions (temperature, vibration etc.), display 
mechanical interface to the user's system, and integrate all other design requirements to provide a suitable and compact mechanical design.
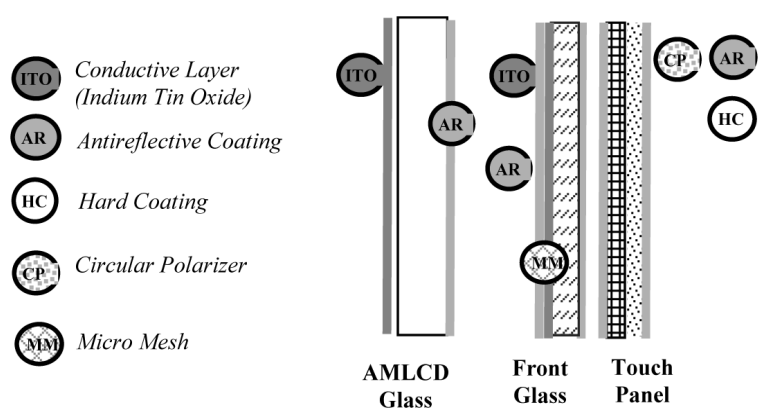

Fig. 5. AFV display cover glass structure

Electrical/Hardware design: provides electrical power interface design (external and internal), EMI/ EMC compatibility, AMLCD driving and control circuits, LED backlight driving circuits, backlight control functions, colour sensor and bezel illumination sensor (which are usual in avionics but also possible to be effective in AFV vetronics), temperature sensor, etc.

Software (firmware) design: involves custom design creations leading to an extended range of the display microprocessor controlled functions, required video signal interface and transformation functions, Built-in Test functions (BIT), etc.

Optical design: usually involves design solutions to include a more reliable, high output, wide dimming range LED backlight, and special display optical features such as: NVIS compatibility, high ambient illumination readability, high brightness, high contrast under illumination, etc. Important part of optical design is COTS AMLCD panel selection. All engineering solutions are trade-offs.

As depicted in Fig. 4, a ruggedised display is a complex optoelectronic system, which requires a complex design process joining various technological solutions into unique equipment. The display key subsystems are described below.

Bezel: incorporates switches, pushbuttons and indicators which can be equipped with appropriate backlight including NVIS filtered backlight. Also, ambient light sensors could be used as a part of the display brightness automatic control electronics, as a possibility required by customers.

Housing, mechanical interface: used to provide display parts integration and proper mechanical mounting in the user's system.

Ruggedised display device: is the display system's most important part. It integrates the display panel and other technologies into a unique functionality. Generally speaking this part consists of:

- touch panel (if required),

- front (cover) glass (Fig. 5), providing vandal protection, anti-reflective front surface (if a touch screen is not used), transparent conductive EMI layer, spectral filtering for contrast enhancement and/or NVIS, could be also as the mesh solution to provide better screening,

- display device (CRT, FEL, AMLCD, OLED) electronically controlled pixelated structure,

- display device heater (for the LCD option), providing low temperature operation.

Backlight: is another critical subsystem (if used) consisting of two main parts:

a. optical stack (light collection and beam shaping optics, with possible including of DBEF film for efficiency improving) and

b. illumination source (suitable light source, CCFL, White LEDs, RGB LEDs).

The backlight could be designed as:

- bottom - direct illuminated (illumination source is distributed inside light integration chamber bellow AMCLD active surface),

- side illuminated (illumination source is suited on the side and light is transferred to the AMLCD using waveguide optics).

Backlight driving electronics: energy efficient driving circuits including dimming control electronics, of wide range.

Display driving electronics: drives display pixels (light valves) according to video signal content, as the integral part of LCD cell externally controlled.

Motherboard \& microcontroller: integrates all electronics and control circuits.

Software and firmware: application tailored embedded software and firmware controlling display functions and controls.

Communication interface electronics electronic boards designed for proper communication with user system.

Power interface: transform external power source voltage to voltages needed for display operation, according to different military standards.

Video signal interface electronics: transforms used video signal formats to the signal compatible with AMLCD driving electronics.

Sensors: usually used to sense display temperature, illumination level or colour, and produce signals used in the display automatic control functions. 


\section{DISPLAY BASIC PROPERTIES}

The main purpose of any display is to show visual information (for human visual system, HVS). Display parameters should be controllable, defined to provide unambiguous requirements of display suitability in intended application.

It is possible to define suitable display characteristics and performances. A specific application needs specific requirements and measurement methods.

Information capacity. The amount of visual "information" that a display is able to convey is related to the "information content" of a display. It is defined by the total number of pixels, the size of the pixels (resolution), colour, the number of grey levels and the size of the display. On the other hand, there is the eye's ability to discern details - our visual acuity [16] and limitations of human perception.

The acuity of an average human eye can resolve an individual pixel of approximately one arcminute of the visual context wide, with additional conditions attached. Visual acuity determines the level of detail that an eye can absorb from the pattern of pixels present on a screen. The closer the object being viewed, the smaller the level of detail can be determined.

Brightness (Luminance). The brightness of a viewed object is defined in a psychological sense as the level of light intensity perceived by a viewer. The key physical measure of brightness is luminance. Brightness is defined as the luminance of white colour in the centre of the screen and is measured in candela per square metre $\left(\mathrm{cd} / \mathrm{m}^{2}=\right.$ nit $)$ or foot-lamberts $(1 \mathrm{fL}=$ 3.426 nits).

The display luminance value required for comfort viewing varies from $100 \mathrm{~cd} / \mathrm{m}^{2}$ in a shadowed office environment up to $1000 \mathrm{~cd} / \mathrm{m}^{2}$ in high ambient illumination environment which need not be the threshold quality factor.

Typical AFV display maximal luminance value is up to $500 \mathrm{~cd} / \mathrm{m}^{2}$.

For display technologies the other important consideration is the luminance dynamic range (dimming range), that is, the ratio between the minimum and maximum luminance that can be generated, and allow display luminance to set value in accordance with human eye accommodation properties. AFV displays have dimming typical range up to 1:200 in day light operating condition and similarly in night operating conditions, if applied.

Contrast. Display contrast ratio is the ratio of maximal luminance to minimal luminance that can be generated in the same point in image. Display contrast is created by the difference in luminance from two adjacent surfaces. It is related to display image detail luminance $(L)$ and background luminance $(L b)$ (usually defined as: $(L-L b) / L b)$ ).

These parameters should be specified in a predefined illumination environment where ambient light and reflections from the screen will significantly affect the values.

A display may not be able to deliver a "pure" black because the technology applied leaks light or reflects ambient light. A good display will offer a contrast ratio that exceeds 1000:1 measured in dark. HVS ability to see details at appropriate contrast levels (contrast sensitivity), required contrast which vary around 50, but higher values provides better reproduction of black, proportional to the grey shades values.

Contrast ratio greater than $5: 1$ is required as the minimum for image details detection in the high ambient light environment usually sufficient as 5.66:1, depending on the mission. In this case black and other colours are "washed up".

Colour Properties. An average human eye can perceive millions of different colours. The 1931 Commission Internationale de l'Eclairage (CIE) developed a three dimensional colour "space" that allowed any visible colour to be mapped. Any colour could be located within the colour space and its composition from each of the three primaries (Red, Green and Blue) can also be determined.

Display colour reproduction ability depends on the quality of display primary colours. It is usually represented by a triangle in the colour space having red, green and blue colour in the corners, also known as the colour gamut. Display gamut is compared with standard gamut (NTSC) and gamut quality is defined as ratio of display gamut area and standard gamut area in (u', v' - CIE 1976) chromaticity chart.

Emissive colour displays usually have more pure primaries and hence wider gamut that non-emissive. Trade-off between LED's emission characteristics and colour filters is also important as the issue of this technology, but beyond the contents of this paper.

Resolution. The key measure for display quality, in accordance with HVS acuity is the pixels density expressed in pixel per inch (PPI) or pixel per millimetre (PPMM). This depends on the available components and customer requirements. The requirements for display resolution depend on the application through anticipated observer to display distance. Some typical pixel densities are: 
- 300 PPI (12 PPMM) is typical visual acuity limit for hand held device displays (retinal displays),

- 170 PPI (7 PPMM) visual acuity limit for displays is the best in the avionics technology as a comparison, but not required for vetronics,

- 200 PPI (8 PPMM) is a good approximation of the HVS requirement within a computer graphical display.

Display resolution 100 to $150 \mathrm{PPI}$, is sufficient for most AFV display applications.

Active Area. Active area is a display surface where information content is presented. It is measured by diagonal (usually expressed in inches or $\mathrm{mm}$ ), and aspect ratio (optional technology possible as ratio $1: 1,5: 4,4: 3,16: 10,16: 9)$. AFV mostly displays use standard $4: 3$ aspect ratio, but different size pending on application. Usually, it is not necessary to resize COTS AMLCD panels. The most common COTS display size and resolution considered for AFV display applications are presented in Table 1.

Table 1. AFV Display Commonly used size and resolution

\begin{tabular}{|c|c|c|c|c|c|}
\hline \multirow{2}{*}{ Diagonal } & \multicolumn{3}{|c|}{ Resolution } & \multicolumn{2}{|c|}{ Orientation } \\
\hline & VGA & SVGA & XGA & Land. & Port. \\
\hline 5" $(12.7 \mathrm{~cm})$ & - & & & • & \\
\hline $5.7 "(14.5 \mathrm{~cm})$ & $\bullet$ & & & $\bullet$ & \\
\hline $6.5 "(16.5 \mathrm{~cm})$ & $\bullet$ & & $\bullet$ & $\bullet$ & \\
\hline $8.4 "(21.3 \mathrm{~cm})$ & & $\bullet$ & $\bullet$ & - & $\cdot$ \\
\hline $10.4 "(26.4 \mathrm{~cm})$ & & $\bullet$ & $\bullet$ & $\bullet$ & $\bullet$ \\
\hline $12.1 "(30.7 \mathrm{~cm})$ & & & $\cdot$ & $\bullet$ & \\
\hline VGA $(6140 \times 480)$ & $\mathrm{GA}$ & $600)$ & $(10$ & 68) & \\
\hline
\end{tabular}

There are a variety of COTS panels suitable for ruggedisation. Some of them are successfully applied in current designs. The 10.4" $(26.4 \mathrm{~cm})$ XGA AMLCD display is dominant in current MFD applications. Proper display size availability is system designer issue, and is limited by AFV.

Viewing Envelope. The angle of view is defined as the angle at which the viewer is positioned in relation to the screen in order to clearly see the whole image on a display. The angular viewing envelope is the space that includes all the required viewing angles. AFV display usually has a viewing envelope that normally covers at least $\pm 60^{\circ}$ horizontally and $+30^{\circ}$ \% $-20^{\circ}$ vertically, but more specific requirements could be set according to application.

Response-time. The time an individual pixel or cell in a display screen takes to change from open to close and reverse, is known as the response time and is measured in milliseconds, [ms]. The response time affects the ability to change an image rapidly on the screen.
Some typical response time values required are:

- $25 \mathrm{~ms}$ for general computer applications,

- 12 to $15 \mathrm{~ms}$ for TV, sports and gaming,

- $100 \mathrm{~ms}$ in the case of AFV display displaying graphical content.

The above mentioned cases could be satisfactory for the slow changing graphic content.

LCD has an inherent latency time due to the switching of the liquid crystal, which introduces a longer response time than is required by specific applications. Also, switching time highly depends on temperature. To operate at low temperatures LCD requires uniform heating.

Sun-Readability. AFV display typical requirement is readability in a high ambient lighting from a low as zero to a high as 10,000 to $50,000 \mathrm{~lx}$ of diffuse and/or collimated illumination from one or more directions. The display should be mounted to minimize the impact of the ambient illumination, which is hard to achieve. The display front end should be optically enhanced to minimize diffuse and specular reflectance.

For "mission critical" display a high ambient illumination requirement is usually specified through threshold contrast ratio [18] in given illumination environment (Collimated source generating illumination up to 50,000 $\mathrm{lx}(5,000 \mathrm{fc})$, wide diffusive source with luminance up to $6,800 \mathrm{~cd} / \mathrm{m}^{2}(2,000 \mathrm{fL})$ ).

The measurement method and set-up should be clearly defined. The minimally required contrast value depends on type of information displayed (2 - alpha numerical; 3 - graphics; 4.66 (6 $\sqrt{2}$ grey levels) for $\mathrm{B} / \mathrm{W}$ image) [17].

NVIS Compatibility. To obtain the possibility to use both display unit and NVG (Night Vision Goggle) at the same time (night driving), a specific technical solution is selected. By using special optical filters on NVG and display unit, the haring of optical spectra is achieved. NVG are filtered using appropriate filters, and a display unit is filtered to eliminate excess of NIR radiation, so the display can be operated successfully using the naked eye, and without disturbing NVG [18]. A display used in NVIS compatible mode has limited gamut ("poor" reproduction of red).

In addition, the display should not disturb the crew NVG during night operations. Also, secure lighting generates additional requirements.

Electromagnetic Compatibility. To provide proper EMC display electronics are equipped with EMI filters, and display active area is covered with a transparent conductive layer (ITO or micro mesh). 
Environmental Properties. A wide operation temperature range is the most critical environmental requirement.

Operation at low temperatures (down to $-46{ }^{\circ} \mathrm{C}$ ) could be achieved by using heaters, but operation at high temperatures should be inherent to display technology. Some of the COTS AMLCD panels are tested at extreme operation temperature range from -30 to $+80{ }^{\circ} \mathrm{C}$ and storage temperature range -40 to $+85^{\circ} \mathrm{C}$, but less range also has enough good reserve tolerance fields.

\section{DISPLAY TECHNOLOGIES}

The simplified classification of the flat panel display technologies is shown in Fig. 6.

An emissive display is one that produces its own light; a passive (non-emissive) display modulates light that passes through it.

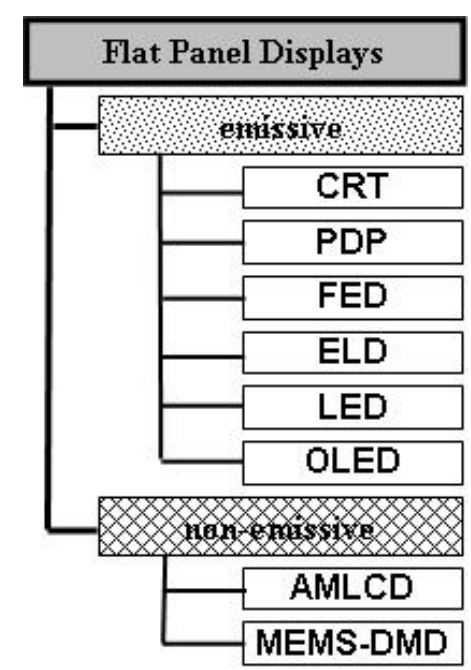

Fig. 6. Flat Panel Display classification

\subsection{Emissive Display Technologies}

CRT, Cathode Ray Tube [16]. CRT are not suitable for the AFV applications because they are expensive, not usually available and too large. CRT technology has a historical role but is not considered as suitable for new designs.

FED, Field Emission Displays. FED use electrons to directly fire-up a phosphor screen, in the same manner as traditional CRTs. However, with field emission devices, the mechanism used to generate the electrons is completely different. It is non-thermion and uses the physical properties of "field emission effect" and "quantum tunnelling", whereby a low voltage is applied to a very large number of tiny, highly pointed cathodes in order to release electrons. These cathodes can be made from a number of possible materials including carbon inks, diamond-based structures, Spindt tips (molybdenum) and carbon nanotubes. Carbon nanotubes are one of the new products emerging from the field of nanotechnology. FED technology is an interesting solution but still not close to mass production. This technology is not available on the commercial market yet, but it is expected to be soon.

LED, Light Emitting Diode. LED light intensity is proportional to the bias current and the colour dependent on the material used. LED display units are based on matrix of individual emitters. To achieve small pixel size is hard and the production process is very expensive. LEDs are commonly used in backlights and for small dedicated function displays.

ELD, Electro-Luminescent Displays. A phosphor film between glass plates emits light when an electric field is created across the film, and is known as the base for the so called ELD technology [20]. This technology type is effectively singlesourced, monochrome, difficult to obtain video and power hungry - an obsolete technology.

PDP, Plasma Displays. Plasma screens are composed of millions of cells sandwiched between two panels of glass.

PDP technology is suitable for high display size (diagonal higher than 32 " $(81.3 \mathrm{~cm})$ ), so there is no application in AFV displays.

OLED, Organic Light Emitting Diode. OLEDs use a very thin film of an organic substance that can emit red, green, blue or white light when a charge is applied. Display devices are made up of layers of this organic material sandwiched between a positive (anode) layer and a negative (cathode) layer. OLED technology has the potential for large-scale production using printing processes and it is believed it would overcome some of the limitations of LCD [21]. These characteristics, particularly the low power consumption, made OLEDs as promising for smaller display screens and they are already being used in handheld devices.

\subsection{Non-Emissive Display Technologies}

LCD, Liquid Crystal. A liquid crystal material, acting likes a shutter: blocks, dims, or passes light unobstructed, depending on the magnitude of the electric field across the material. It is used in connection with the backlight providing controlled light output from display. 
To form a working LCD individual components (glass casing, liquid crystal cell, alignment layer, conductive electrodes, and polarizer) are combined. The light entering the display is polarised by back polarizer, and interact with oriented liquid crystal molecules. When voltage is applied, the liquid crystal molecules orientation is changed causing the liquid crystal polarisation properties. As a result of interaction with second polarizer the luminance of the output light could to vary from minimal to maximal value pending on voltage applied. A colour filter is applied to provide colour sub-pixel.

High-end displays today easily have 256 different levels of light or shades allowing a grey scale range in which graphics and characters can be displayed in many varying intensities [19].

AMLCD Basic Technologies defining the AMLCD panel structure and functioning are Twisted Nematic (TN), - Super Twisted Nematic (STN), Vertical Alignment (VA), Multidomain Vertical Alignment, (MVA) In Plane Switching (IPS), Advanced Field Fringe Switching (AFFS).

TN (STN) displays have a narrow viewing envelope. To achieve a wide viewing angle, additional optical compensation films are used.

VA and IPS technology provides wide viewing angle.

Micro Electro Mechanical Systems (MEMS) and Projection Display. MEMS are miniature devices, which integrate actuators, sensors, and processors to form intelligent systems. Functional optical sub-systems controls light transmission or reflection. There are few emerging DLP technologies still in development (Grating valve display, Pixtronix MEMS shutter with Field sequential RGB backlight, Mirasol displays based on bi-stable interferometric modulation).

Combined with a digital video or graphic signal, a light source and a projection lens, the mirrors of the DMD chip can reflect an all-digital image onto any surface.

DLP Projection displays are too bulky for AFV applications. Other MEMS display technologies are not ready for mass production and application.

\subsection{Ruggedisation Technologies}

There are various ruggedisation techniques applicable to enhance display properties, such as:

Optical Bonding. One of the most important is optical bonding that allows us to join different layers (glass, filters, or films) to enhance display rigidity and display front end optical or conductive properties.
The resultant assembly is very rugged, shock, impact and vibration resistant and maximizing optical properties at the same time. Two main technologies used for optical bonding are: (i) liquid bonding (using silicones, epoxies, polyurethane) - Optically Clear Adhesive (OCA) and (ii) dry bonding (using roll on process based on application of pressure and heat activated dry adhesive sheets.

Resizing. To allow that AMLCD COTS displays could be used in applications requiring specific shape, resizing process has been applied. This is rather complicated [22] and [23], and protected with patents. Resizing has an important role in AMLCD technology application mainly in cockpits, but could be used in any other application.

EMI Shielding. EMI interference could be critical in the AFV environment. There are several techniques suitable and proved for EMI shielding optimization: (i) EMI filtering on the PCB; (ii) proper grounding techniques; (iii) transparent conductive layer over display active surface (ITO or micromesh), forming proper Faraday cage together with metal housing.

\subsection{Touch Panel Technologies}

Touch Panel - Screen is a position-sensitive device, which could be activated using finger or stylus. At the same time, they are transmissive and could be overlaid $t$ display active surface. This allows integration of the display visual content and touch position detection into unique human machine interface capability. This capability makes them very suitable as data input device for mobile applications.

To be considered as suitable for integration, touch panels should have sufficiently high optical clarity, transmission and touch position sensing resolution.

The major benefits of the touch technologies are: (i) easy to use - what you see you touch to generate command, (ii) flexible - Using the same interface one can implement different options, (iii) upgradeable - easy and fast changes through software, (iv) cost effective - this is a relative issue depending on application, (v) rugged and reliable performances - could be considered for use in an extremely harsh environment.

Having in mind the promising capabilities, a lot of efforts have been made in the development of suitable technologies. Some of them are applicable in AFV displays:

- Resistive (RES),

- Capacitive \& Projective Capacitive (CAP and PCAP), 
- $\quad$ Surface Acoustic Wave (SAW),

- Infrared Array Sensor (IR).

A comparison of applicability properties of touch screen technologies in military environment is shown in Table 2.

Table 2. Touch Screen Technology Comparison

\begin{tabular}{|c|c|c|c|c|}
\hline \multirow[t]{2}{*}{ Benefits } & \multicolumn{4}{|c|}{ Touch panel technology } \\
\hline & IR & RES & CAP & SAW \\
\hline Vandal Resistant & $\bullet \bullet$ & $\bullet$ & $\bullet$ & $\bullet$ \\
\hline Scratch Resistant & $\bullet \bullet$ & $\bullet$ & $\bullet$ & $\bullet$ \\
\hline Not sensitive to dirt & $\bullet$ & $\cdots$ & $\bullet$ & $\cdot$ \\
\hline Non Sensitive to EMI & $\bullet \bullet$ & $\cdots$ & $\bullet$ & $\cdot$ \\
\hline Can be FINGRER operated & $\bullet \bullet$ & $\bullet \bullet$ & $\bullet$ & $\bullet$ \\
\hline Can be GLOVE operated & $\bullet \bullet$ & $\bullet$ & $\bullet$ & $\bullet$ \\
\hline Can be hermetically sealed & $\bullet \bullet$ & $\cdots$ & $\cdots$ & $\cdots$ \\
\hline Sensitivity to temperature & $\bullet \bullet$ & $\bullet$ & $\bullet \bullet$ & $\bullet \bullet$ \\
\hline Suit military environment & $\bullet \bullet$ & $\bullet$ & $\bullet \bullet$ & $\bullet \bullet$ \\
\hline Integration & $\bullet \bullet$ & $\bullet$ & $\bullet \bullet$ & $\cdot$ \\
\hline NVIS compatibility & $\bullet$ & $\cdots$ & $\cdots$ & $\bullet \bullet$ \\
\hline
\end{tabular}

\section{AFV DISPLAY TECHNOLOGY EVOLUTIONS}

CRTs were used in AFV fire control systems to display sensor (TV or thermal imager) image integrated with reticule and other sighting related data.

Multifunctional and moving-map displays were the first objectives which generated new developments using any new technology available.

Electroluminescence panels were introduced as the first all solid state based displays, well suited to AFV environment. Relatively low brightness (about $50 \mathrm{fL}$ as the extreme values probably reduced on the half by filtering) high panel production price together with lack of colour TFEL in mass production were the key disadvantages that contributed to their low application as future AFV displays.

Introduction of AMLCD for avionics in the mid 1980s, as feasible technology was an example how these technologies cold find application in the demanding environments as aircrafts and AFVs are. After that AMLCD application spread in all other military applications, including AFV related displays.

In the 1990s, display devices research and development were accelerated and long term development road-map was set down [14]. AMLCD panel storage, start up were the less problems but the main have been operating temperature.

The LC materials remain operable, although slower, down to $-30^{\circ} \mathrm{C}$ and lower. Speed of operation can be increased with heating via a transparent Indium-
Tin-Oxide (ITO) thin-film heater applied close to the AMLCD panel, although not very effective regarding that some sources use non-transparent heater behind BLU. Also, the LED backlight acts as a heater because of its power dissipation contributing to panel surface temperature increase up to $10^{\circ} \mathrm{C}$.

The high operation temperature is limited by the clearance point of the LC material. Above the clearance point temperature, an immediate but reversible loss of image occurs. Modern LC materials can be fabricated with a clearance point temperature of over $100{ }^{\circ} \mathrm{C}$ without sacrificing other properties, with possibility to trade values vs response time.

The back-lit AMLCD is unique in several ways that prove to be highly desirable attributes for AFV use. The following objectives for AFV displaying instruments are satisfied by the AMLCD:

i. Low volume with low weight and power preferably without forced air cooling;

ii. Wide luminance range is easily achievable (from $0.1713 \mathrm{~cd} / \mathrm{m}^{2}$ to as high as $685.2 \mathrm{~cd} / \mathrm{m}^{2}$ );

iii. Colour primaries of saturated red, green and blue with up to 256 shades of grey in each primary, but usually taken as lower values of 64 shades.

iv. Wide viewing angle greater than $\pm 60^{\circ}$ in both horizontal and vertical direction, also variable by trade-off in vertical direction.

v. Readability in high ambient illumination with contrast ratios of at least five to one. This depends on the internal design and the definition of high ambient illumination.

vi. Sufficiently uniform brightness through the image plane without distortion, and acceptable resolution with at 100 to 150 PPI pixels density, variable by supplier's options and cost.

vii. Response speed suitable for frame rates up to 80 frames per second.

\section{CONCLUSIONS}

AFV vetronics systems are still not fully defined, but results from current research could be used in AFV upgrade programs, as well as for future hybrid electric heavy duty vehicles [24].

AFV display technologies are limited only to what the COTS sources can supply. Being the elements of ubiquitous computing systems [25], they allow AFV system integrator to specify proper requirements delivering desirable HMI functions.

CRT displays were only used for essential video sighting functions. They introduced image application in AFV control stations increasing display information capacity. 
ELD (TFEL) and LED displays find their place in some specific application opening the door to all solid state displays.

Back-lit AMLCD shows up as ideal for application in AFV systems. Nowadays, there is no display technology that comes close to compete with AMLCD availability, performance and price.

Liquid crystal development forced by TV related application, contributed to increase LCD speed and viewing envelope. Hand held and industrial application contributed more or less to increase in clearance temperature.

AMLCD market is fast evolving in the area of mobile displays, computer and laptop monitors, but it is stable in the area of industrial and special application displays. AFV display suitable sizes and resolutions are possible to be covered with industrial display panels, depending on commissioner.

The AMOLED displays are a new and competitive technology, having mass production for small area mobile displays, and emerging application as TV displays, but it will not be ready for mass production for sizes suitable for AFV vetronics systems in the next five years. The first application of this technology could be for personal AFV crew displays.

Resistive touch screen has some applications in AFV displays, but there are issues with life time. Also, there is a need for more research regarding human factors affecting their effective usage as HMI device in military environment.

AFV vetronics systems designers could count on COTS AMLCD panels and ruggedisation process to provide suitable AFV displays in various Crew Station configurations.

There are new display technologies under development but it seems that none is ready enough to replace AMLCD technology in AFV application during this decade.

\section{ACKNOWLEDGEMENT}

This work has been done within the project III 47029 supported by Ministry of Science and Technological Development of the Republic of Serbia.

\section{REFERENCES}

[1] Smith, E. (2001). Effects Based Operations: Applying Network Centric Warfare in Peace, Crisis, and War. CCRP, Washington.

[2] Alberts, D., Garstka, J., Stein, F. (1999). Network Centric Warfare: Developing and Leveraging Information Superiority. CCRP, Washington.
[3] Edwards, S. (2005). Swarming and the Future of Warfare. Rand Corporation, Santa Monica.

[4] Janković, R. (2011). Computer Simulation of an Armoured Battalion Swarming. Defence Science Journal, vol. 61, no. 1, p. 36-43.

[5] Janković, R., Milinović, M., Nikolić, N., Jeremić, O. (2011). On application of discrete event simulation in armoured and mechanized units research. Proceedings of $1^{\text {st }}$ International Symposium and $10^{\text {th }}$ Balkan Conference on Operational Research, Thessalonica.

[6] STANAG 2895 (1990). Extreme climatic conditions and derived conditions for use in defining design/test criteria for NATO forces materiel, Edition 1, NATO Military Agency for standardization, Brussels.

[7] MIL-STD-810G (2008). Department of Defence Test Method Standard for Environmental Engineering Considerations and Laboratory Tests. US Department of Defence, Washington.

[8] Downs, G., Roller, G., Brendle, B.E., Tierney, T. (2000). Military application of flat panel displays in the vetronics technology test bed prototype vehicle. Proceedings SPIE, vol. 4022 - Cockpit Displays VII: Displays for Defence Application, p. 75-84, DOI:10.1117/12.397776.

[9] Thomsen, S., Weindorf, P. (1999). AMLCD Design Considerations for Avionics and Vetronics Applications. Proceedings SPIE, vol. 3363 - Cockpit Displays VII: Displays for Defence Application, p. 4860, DOI:10.1117/12.321807.

[10] Busse, D. (1999). Display integration for ground combat vehicles. Proceedings SPIE, vol. 3363 - Cockpit Displays VII: Displays for Defence Application, p. 151162, DOI:10.1117/12.321769.

[11] Downs, G. (2001). Vetronics technology demonstrator display technology. Proceedings from 2001 Vehicle Technologies Symposium - Intelligent Systems for the Objective Force, document ADA393833.

[12] Dowling, S. (2004). Vetronics in the UK and Europe. Cranfield University, Cranfield.

[13] Dowling, S. (2008). Vetronics architectures. Proceedings of Design Automation and Test in Europe, from http://groups.engin.umd.umich.edu/vi/w5 workshops/dowling_Vetronics\%20Architectures.pdf, accessed on 2012-05-25.

[14] Hopper, D. (2000). High resolution displays and roadmap. Proceedings of the 10th International Conference on Artificial Reality and Tele-existence, Taipei.

[15] Hopper, D., Desjardins, D. (2004). 6.1: Military Cockpit Display Performance Requirements. ADEAC.

[16] Wisnieff, R., Ritsko, J. (2000). Electronic displays for information technology. IBM Journal of Research and Development, vol. 44, no. 3, p. 409-422, DOI:10.1147/ rd.443.0409.

[17] MIL-HDBK-87213A (2005). Electronically / optically generated airborne displays. US Department of Defence, Washington. 
[18] MIL-STD-3009 (2001). Lighting, aircraft, night vision imaging system (NVIS) compatible. US Department of Defence, Washington.

[19] Der Boer, W. (2005). Active Matrix Liquid Crystal Displays: Fundamentals and Applications. Elsevier Inc., Burlington.

[20] Krasnov, A. (2003). Electroluminescent displays: history and lessons learned. Displays, vol. 24, p. 73-79, DOI:10.1016/S0141-9382(03)00015-5.

[21] Zyung, T., Kim, S.H., Chu, H.Y., Lee, J.H., Lim, S.C., Lee, J.-I., Oh, J. (2005). Flexible Organic LED and Organic Thin-Film Transistor. Proceedings of the IEEE, vol. 93, no. 7, p. 1265-1272, DOI:10.1109/ JPROC.2005.850303.
[22] U.S. Patent No. 7,161,651 B2 (2007). Method of resizing a liquid crystal display. U.S. Patent and Trademark, Alexandria.

[23] U.S. Patent No. 6,509,949 (2003). Method of resizing a liquid crystal display. U.S. Patent and Trademark, Alexandria.

[24] Katrašnik, T. (2010). Fuel economy of hybrid electric heavy duty vehicles. Strojniški vestnik - Journal of Mechanical Engineering, vol. 56, no. 12, p. 791-802.

[25] Gerritsen, B., Horwáth, I. (2010). The upcoming and proliferation of ubiquitous technologies in products and processes. Strojniški vestnik - Journal of Mechanical Engineering, vol. 56, no. 11, p. 765-783. 